\title{
Design and Fabrication of a MEMS Capacitive Chemical Sensor System
}

\author{
Vishal Saxena, Todd J. Plum, Jeff R. Jessing and R. Jacob Baker \\ ECE Dept., Boise State University, vishalsaxena@ieee.org
}

\begin{abstract}
This paper describes the development of a MEMS sensor system to detect volatile compounds. The sensor consists of a MEMS capacitive sensor element monolithically integrated with a sensing circuit. The sensor element is a parallel plate capacitor using a chemically sensitive polymer as the dielectric. In presence of the target analyte, the polymer swells and changes the capacitance of the sensor element. This change in capacitance is sensed and converted to a digital bit stream by a delta-sigma sensing circuit. This paper provides an overview of the design of the sensor element, the sensing circuit and the process integration for their fabrication on a single die.
\end{abstract}

Keywords-MEMS Chemical Sensors, Chemicapacitive Sensors, Delta-sigma Sensing, Fabrication, Process Integration.

\section{INTRODUCTION}

$\mathrm{C}$ Themical sensors are being widely deployed for environmental monitoring, industrial hazard detection and sensing of chemical warfare agents. Micro-electromechanical systems (MEMS) technology has enabled design of miniaturized and fully integrated chemical sensors. MEMS chemical sensors detect presence of chemical analytes and produce electrical responses proportional to the concentration of the target analyte. These electrical responses are detected and amplified using a sensing circuit which is typically fabricated using CMOS (Complementary Metal Oxide Semiconductor) process. Monolithic integration of MEMS sensors and interface circuits results in a low power, autonomous, miniaturized, and reliable sensor system-on-achip $(\mathrm{SoC})$ [1]. This sensor $\mathrm{SoC}$ can be easily extended to include an RF subsystem to construct self-sustained wireless sensor networks [2].

This paper presents the development of a MEMS capacitive chemical sensor system. The following sections describe the sensor system architecture, design of the MEMS sensor element and sensing circuit, and their fabrication.

\section{CAPACITIVE MEMS SENSOR SYSTEM}

The capacitive MEMS sensor system (fig.1) detects volatile analytes by producing a change in capacitance, proportional to the target analyte concentration.

The sensing circuit converts the change in capacitance to a voltage signal. The sensed voltage is converted to a digital signal, which is output to an external logic block for data processing. The MEMS sensor element and the sensing circuit are both to be integrated on the same die.

Support of this project by EPA Contract No. X-97031101-0 is gratefully acknowledged.

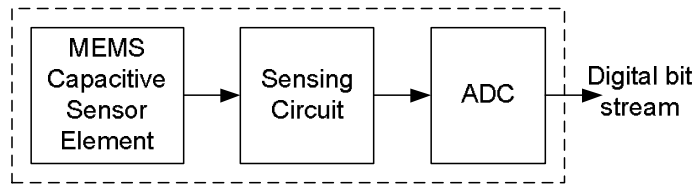

Figure 1. Block diagram of the chemical sensor system.

\section{MEMS SENSOR ELEMENT}

The sensor element is designed as a parallel-plate capacitor composed of overlapping metal layers with a chemically sensitive polymer used as the dielectric (fig.2). Neglecting the fringe capacitance, the sensor capacitance is given as $C_{s}=\varepsilon_{\text {poly }} . A / t$, where $\varepsilon_{\text {poly }}$ is the net dielectric permittivity of the polymer, ' $t$ ' is the mean polymer thickness and ' $A$ ' ' is the metal plates overlap area.

When the sensor is exposed to the target analyte, absorption from the gaseous phase into the bulk of polymer takes place. It results in swelling in the polymer (i.e. $t$ increases) and an increase in dielectric permittivity $\left(\varepsilon_{\text {poly }}\right)$. These changes in sensor property change the sensor capacitance as $\delta C_{s} / C_{s}=\delta \varepsilon / \varepsilon-\delta t / t[1]$.

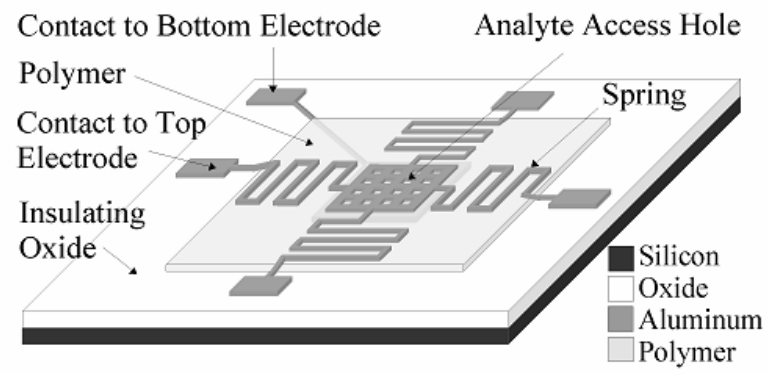

Figure 2. A MEMS capacitive sensor element [3].

The effects of polymer swelling and change in permittivity, on the change in the sensor capacitance, counter each other and may mutually cancel. To avoid this situation the polymer is carefully chosen such that the polymer swelling is maximized for the target analyte. The permittivity of the selected polymer should be as small as possible for maximum sensitivity. The capacitive sensor designed in this work relies upon the polymer swelling as the dominant effect. The polymer which is being experimented in the first sensor prototype is poly-ethylene vinyl-acetate (PEVA) which swells on exposure to benzene [3]. 


\section{SENSOR INTERFACE CIRCUIT}

For circuit design, the capacitive MEMS sensor element is modeled as a variable capacitor. A MEMS capacitive sensor design is reported in [4], which utilizes the change in permittivity of the polymer dielectric upon exposure, to sense the target analyte. The base capacitance of this sensor is around $10 \mathrm{pF}$ and the incremental change in capacitance is $4 \mathrm{aF} / \mathrm{ppm}$. In order to sense such small variations, a high resolution and noise tolerant sensing circuit is required.

A delta-sigma modulation based sensing circuit topology is ideal for this application as it provides high-resolution, noise tolerance, high linearity and compact circuit integration. The advantage of employing delta-sigma sensing is that the analog to digital converter (ADC) is inherently realized in the topology [4].

Fig.3 illustrates a simple first order delta-sigma (oversampling) sensing topology which uses feedback to balance the charge flows from the sensor capacitor $C_{s}$ and reference capacitor $C_{r e f}$, to capacitor $C_{f}$. The circuit uses non-overlapping clocks $\varphi_{1}$ and $\varphi_{2}$. The reference capacitor is identical to the sensor capacitor, except that is hermetically sealed to avoid exposure to the analyte. The reference capacitor is used for differential sensing which eliminates the effects of aging i.e. change in sensor properties with time and usage [5], [6].

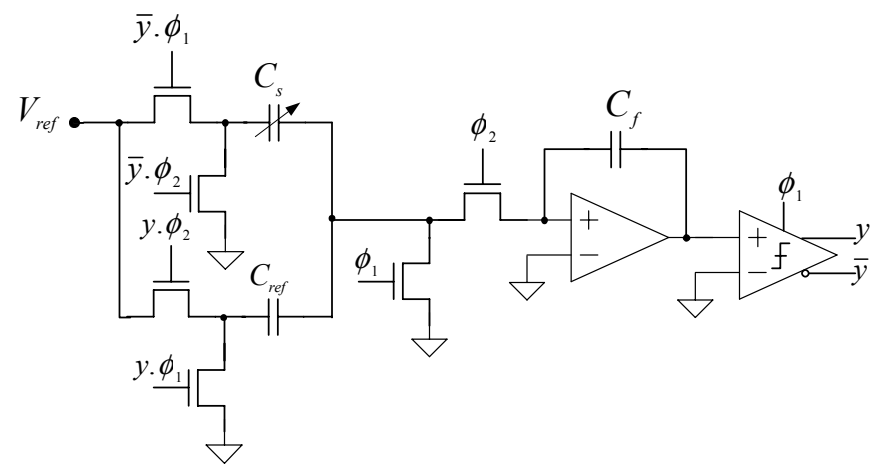

Figure 3. A first order delta-sigma sensing circuit.

The estimate of normalized change in capacitance is given as $\left(C_{s}-C_{r e f}\right) /\left(C_{s}+C_{r e f}\right)=1-2 \hat{y}$, where $\hat{y}$ is the average of the output binary data stream, $y[n]$ [6]. At first, the sensor circuit is designed using only n-type MOSFETs (NMOS) so that it can be fabricated in-house using available NMOS process [7] and easily integrated with the capacitive MEMS sensor.

\section{FABRICATION PROCESS INTEGRATION}

The first sensor prototype is fabricated in the back end of the line (BEOL) of a simple metal-gate NMOS fabrication process, developed at BSU cleanroom [7]. To start with the process, a $5000 \AA$ thick sacrificial oxide is grown on a (100) ptype wafer. This oxide is patterned with 'active' mask to create drain source regions. The drain source regions are doped with phosphorus using spin-on dopant film, followed by a drive-in step. The sacrificial oxide is stripped and $5000 \AA$ thick oxide is grown. This oxide is patterned with 'gate' mask to create gate region windows which are aligned with the source/drains. Then a high quality $200 \AA$ gate oxide is grown using dry oxidation.

The wafer is now patterned using the 'contact' mask to etch contact holes into the field oxide. Then, a $2500 \AA$ aluminum film is sputtered to fill the contact holes and deposit metall layer. This metal layer is pattern with 'metall' mask to create bottom electrode of the sensor and wires for NMOS circuits. After this, an oxide layer is blanket deposited which is patterned with 'vial' mask to open via holes and bottom plate of the sensor element. Next, the chemically sensitive polymer is applied using a spin coater to yield a uniform, $1 \mu \mathrm{m}$ thick film. A 'titanium etch' mask is sputtered and patterned on the polymer. The polymer is then etched in a barrel asher using $\mathrm{O}_{2}$ and $\mathrm{CF}_{4}$ as reagent gases. Finally a $2500 \AA$ aluminum film is sputtered with 'metal2' mask to create the top electrode of the sensor.

\section{PROGRESS AND FUTURE WORK}

MEMS Sensor elements are currently being fabricated. A simple NMOS process for fabrication of sensing circuits has been developed. Presently delta-sigma sensing topologies are being designed, which will be fabricated and tested. Finally the MEMS sensor element will be integrated with the sensing circuit on a single die and tested.

\section{CONCLUSION}

A MEMS capacitive sensor system for sensing volatile chemicals has been proposed. The design and fabrication method for the sensor element and a simple sensing circuit is presented. The MEMS sensor elements are being fabricated. A simple in-house NMOS process is developed for fabrication of sensor interface circuits. After isolated testing of the sensor element and the sensing circuit, both will be monolithically integrated on a single die.

\section{REFERENCES}

[1] A. Hierlmann, Integrated Chemical Microsensor Systems in CMOS Technology, Zurich, Switzerland: Springer-Verlag, 2005.

[2] A. Arora et al, "A Wireless Sensor Network for for Target Detection, Classification, and Tracking," Computer Networks Journal, Oct. 2004.

[3] T. J. Plum, V. Saxena and J.R. Jessing, "Design of a MEMS Capacitive Chemical Sensor Based on Polymer Swelling," submitted to IEEE WMED 2006.

[4] C. Hagleitner, A. Heirlemann, and H. Baltes, "Single-Chip CMOS Capacitive Gas Sensor for Detection of Volatile Organic Compounds," IEEE Sensors Conference 2002, vol. 2, pp. 1428-1431.

[5] R. J. Baker, CMOS: Circuit Design, Layout and Simulation, $2^{\text {nd }}$ ed. Boise, ID: Wiley-IEEE, 2005, chap. 25.

[6] B. Wang, T. Kajita, T. Sun and G. Temes, "High-Accuracy Circuits for On-Chip Capacitive Ratio Testing and Sensor Readout," IEEE Tran. On Instr. And Meas., vol. 47, No. 1, Feb. 1998.

[7] NMOS Fabrication Process, Idaho Microfabrication Laboratory, Boise State University, [Online]. Available:

http://coen.boisestate.edu/imfl/Processes/NMOS_Process.doc 